\section{Optimizing the appeal and benefits of veterinary communication skills training to practitioners, clients, and patients}

\author{
Michael McDermott ${ }^{1,3}$, Malcolm Cobb ${ }^{1}$, \\ lain Robbé ${ }^{2}$, Rachel Dean' \\ 1 University of Nottingham School of Veterinary Medicine and \\ Science, Sutton Bonington, Leicestershire, UK \\ 2 Memorial University of Newfoundland, St. John's, Newfoundland, \\ Canada \\ 3 Premune Animal Health, New York, NY, USA
}

Communication between veterinary surgeons and clients is very important in clinical practice and if done well can positively impact client experience, understanding and compliance, and patient outcomes. The aim of this study was to gain a current understanding of the relevance, impact, and challenges in veterinary communication in practice. A survey of veterinary surgeons was conducted in the United Kingdom
(UK) and United States (US) in 2012 and 2013. A key finding of the study was that $97.7 \%(1,708 / 1,748)$ of respondents felt communication skills were as important if not more important than clinical knowledge. Despite this, only $40.1 \%(705 / 1,759)$ were interested in further postgraduate communication skills training. In an effort to learn more about these seemingly contradictory findings, responses to open-ended questions in the survey were analysed using qualitative research methods (thematic analysis). The key results were: 1) Communication skills should be evaluated beginning with application to veterinary school, given greater attention in veterinary school, and built through a career in practice; 2) In order to increase practitioner willingness to pursue lifelong learning in postgraduate communication skills training, employers should be convinced of its value and communication training should be offered in the most accessible venues and formats for the largest number of veterinary surgeons; and 3) Topics of communication training should directly address the key challenges in communication and the unique needs of practitioners at each stage of their careers. Achieving the above is by no means easy, but would yield significant benefits in practitioner confidence and satisfaction, clients' trust, compliance and loyalty, and perhaps most importantly, quality of care for the patient.

\section{Mortality and relinquishment ascribed to undesirable behaviours in young dogs in the UK}

\section{Caitlin Boyd', Susan Jarvis', Paul McGreevy $^{3}$, Peter Thomson ${ }^{3}$, David Church $^{2}$, Dave Brodbelt ${ }^{2}$, Dan O'Neill ${ }^{2}$

\author{
1 University of Edinburgh, Edinburgh, UK \\ 2 Royal Veterinary College, London, UK \\ 3 The University of Sydney, Sydney, Australia
}

Undesirable behaviours (UBs) are reportedly common in young companion dogs and often reflect compromised welfare, either because they result from sub-optimal prior management or they trigger ill-advised remediation by the owners. Using the VetCompass database of dogs attending primary-care veterinary practices in England, this study explored dogs that either died or were relinquished up to three years of age, to report the prevalence of UBs that led to this outcome and to identify associated risk factors. Descriptive statistics characterised the UB-affected dogs. Univariable logistic regression was used to determine risk factors associated with mortality ascribed to UBs compared with mortality due to non-UBs and relinquishment associated with UB compared with relinquishment for non-UBs.
Of the deceased dogs ( $N=1,421), 36.2 \%$ (95\% Cl: 33.7538.81) died because of UBs. Large breeds (mean breed bodyweight $\geq 20 \mathrm{~kg})$ had $1.25(95 \% \mathrm{Cl}: 1.11-1.67, \mathrm{P}=0.001)$ times the odds of death from UB compared with small breeds and male dogs had 1.5 (95\% Cl: 1.2-1.9, $\mathrm{P}<0.001)$ times the odds of death from UB compared with female dogs. The most common UBs that led to death were aggression (73.97\%) and road traffic accidents (36.7\%).

Of the relinquished dogs ( $\mathrm{N}=307), 24.8 \%$ (95\% Cl: 20.1 30.1) were surrendered due to UBs. The most common UBs causing relinquishment were aggression (46.2\%) and interpet conflict (27.63\%). Large breeds had 1.67 (95\% Cl: 1.11 3.33, $P=0.030$ ) times the odds of relinquishment from UB compared with small breeds and male dogs had 2.2 (95\% $\mathrm{Cl}$ : 1.30-3.70, $\mathrm{P}=0.005)$ times the odds of relinquishment from UB compared with female dogs.

This study identified higher odds of relinquishment and mortality related to UB in large breeds and male dogs compared with small breeds and female dogs, respectively. Aggression was the most common UB associated with both relinquishment and death in dogs. Awareness of these results can assist owners, animal rescue centres and other stakeholders to improve decision-making when rehoming, purchasing and training their dogs. People who purchase large breeds or male dogs may need to consider investing more time in training and enlist assistance from professional sources. These findings could help to reduce the rate of mortality or relinquishment due to UBs. 